\title{
A semirecumbent body position led to a lower rate of nosocomial pneumonia than a supine position in mechanically ventilated adults
}

Drakulovic MB, Torres A, Bauer TT, et al. Supine body position as a risk factor for nosocomial pneumonia in mechanically
ventilated patients: a randomised trial. Lancet 1999 Nov 27;354:1851-8.

QUESTION: In patients who are intubated and mechanically ventilated, is a semirecumbent body position more effective than a supine body position for reducing the incidence of nosocomial pneumonia?

\section{Design}

Randomised (allocation concealed), unblinded, controlled trial with follow up for 72 hours after extubation. An interim analysis was planned.

\section{Setting}

2 intensive care units (ICUs) of a 1000 bed, tertiary care, university hospital in Spain.

\section{Patients}

90 patients (mean age $65 \mathrm{y}, 76 \%$ men) in the ICU who had been intubated and mechanically ventilated. Exclusion criteria were recent abdominal surgery or neurosurgery, shock refractory to vasoactive drugs or volume therapy, or previous endotracheal intubation. $96 \%$ of patients completed the trial.

\section{Intervention}

43 patients were allocated to a semirecumbent body position ( $45^{\circ}$ from the horizontal) and 47 to a supine body position. Patients were fed parenterally or enterally (continuous feeding) at the discretion of the physician. Sucralfate and other medications could be used.

\section{Main outcome measures}

Clinically suspected nosocomial pneumonia. Secondary outcome was microbiological nosocomial pneumonia confirmed by bronchoalveolar lavage or protected specimen brush cultures.

\section{Main results}

The study was stopped early when analysis showed that the semirecumbent position was superior for reductions in clinically suspected pneumonia. Fewer patients in the semirecumbent group developed clinically suspected $(p=0.003)$ or microbiologically confirmed $(p=0.018)$ nosocomial pneumonia than in the supine group (table). Multivariate analysis showed that microbiologically confirmed pneumonia was associated with enteral nutrition (adjusted odds ratio [OR] 11.8, 95\% CI 1.4 to 98) and supine body position (OR 6.1, CI 1.2 to 31). The groups did not differ for mortality $(18 \%$ in the semirecumbent group $v 28 \%$ in the supine group, $\mathrm{p}=0.3$ ).

\section{Conclusion}

For patients in the intensive care unit who were mechanically ventilated, a semirecumbent body position was associated with a lower rate of nosocomial pneumonia than a supine position.

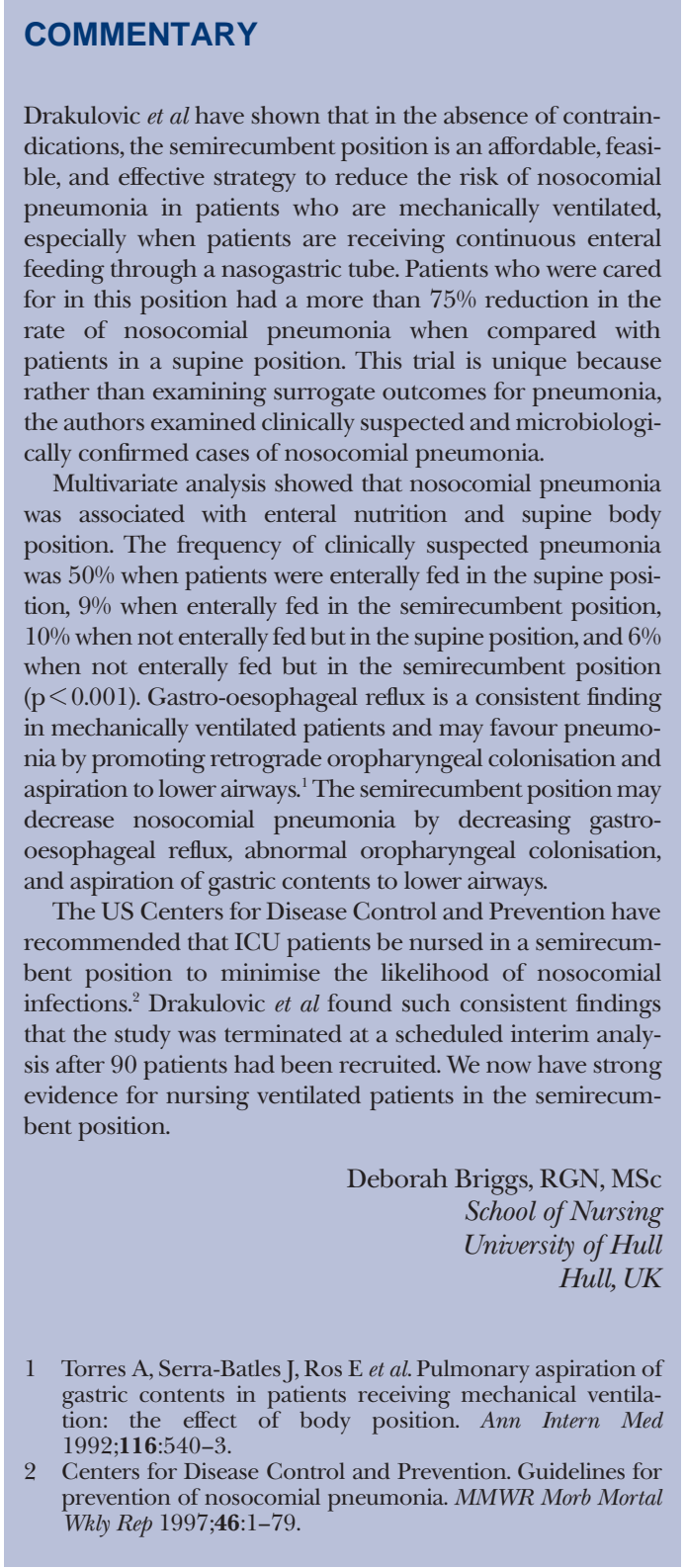

Semirecumbent $v$ supine body position for prevention of nosocomial pneumonia 72 hours after extubation*

\begin{tabular}{|c|c|c|c|c|}
\hline Pneumonia & Semirecumbent & Supine & RRR $(95 \%$ CI) & NNT (CI) \\
\hline Clinically suspected & $8 \%$ & $34 \%$ & $77 \%$ (35 to 93$)$ & 4 (3 to 11$)$ \\
\hline Microbiological & $5 \%$ & $23 \%$ & $78 \%(19$ to 94$)$ & $6(4$ to 29$)$ \\
\hline
\end{tabular}

